\title{
In vivo, pixel-resolution mapping of thick filaments' orientation in nonfibrilar muscle using polarization-sensitive second harmonic generation microscopy
}

\author{
Sotiris Psilodimitrakopoulos \\ Susana I. C. O. Santos \\ Ivan Amat-Roldan \\ Anisha K. N. Thayil \\ ICFO-Institut de Ciencies Fotoniques \\ Mediterranean Technology Park \\ 08860 Castelldefels, Barcelona, Spain
}

\section{David Artigas}

Universitat Politecnica de Catalunya

Department of Signal Theory and Communications 08034 Barcelona, Spain

Pablo Loza-Alvarez

ICFO-Institut de Ciencies Fotoniques Mediterranean Technology Park 08860 Castelldefels, Barcelona, Spain

\begin{abstract}
The polarization dependence of second harmonic generation (SHG) microscopy is used to uncover structural information in different muscle cells in a living Caenorhabditis elegans (C. elegans) nematode. This is done by using a generalized biophysical model in which element ratios for the associated second-order nonlinear tensor and angular orientations for thick filaments are retrieved using a pixelby-pixel fitting algorithm. As a result, multiple arbitrary orientations of thick filaments, at the pixel-resolution level, are revealed in the same image. The validity of our method is first corroborated in wellorganized thick filaments such as the nonfibrilar body wall muscles. Next, a region of the nonstriated muscular cells of the pharynx is analyzed by showing different regions with homogenous orientations of thick filament as well as their radial distribution. As a result, different sets of the nonstriated muscle cell groups in the pharynx of this nematode were exposed. This methodology is presented as a filtering mechanism to uncover biological information unreachable by common intensity SHG microscopy. Finally, a method to experimentally retrieve the distribution of the effective orientation of active SHG molecules is proposed and tested. @ 2009 Society of Photo-Optical Instrumentation Engineers. [DOI: 10.1117/1.3059627]
\end{abstract}

Keywords: microscopy; polarization second harmonic generation; multiphoton processes; biology; scanning microscopy; image analysis.

Paper 08139RR received Apr. 28, 2008; revised manuscript received Nov. 4, 2008; accepted for publication Nov. 10, 2008; published online Jan. 21, 2009.

\section{Introduction}

Second harmonic generation (SHG) imaging is starting to prove to be a very promising tool in biological research. ${ }^{1}$ This is due to the fact that it can provide structural information on biological samples with very high resolution and (compared to other scanning microscopy techniques) in a minimally invasive way. ${ }^{2}$ It relies on the nonlinear interaction of tightly focused ultrashort laser pulses with noncentrosymmetric molecular arrangements, causing scattered coherent radiation at half the fundamental wavelength. ${ }^{1,2}$ The quadratic dependence of the generated signal on the excitation photon flux restricts the phenomenon into a well-defined active volume, which provides the same axial resolution as that of two-photon excited fluorescence (TPEF). ${ }^{3}$ This results in an intrinsic optical sectioning capability, but the possible photodamage is restricted to the focal point, so cell viability is extended. In addition, the use of infrared wavelengths for imaging allows deeper penetration in thick specimens (several hundred microns).

Address all correspondence to Sotiris Psilodimitrakopoulos, ICFO-Institut de Ciencies Fotoniques, Mediterranean Technology Park-Av Canal Olimpic s/n Castelldefels, Barcelona 08860 Spain; Tel: 0034935534071; Fax: 0034935534000; E-mail: sotiris.psilodimitrakopoulos@icfo.es
In contrast to linear and nonlinear fluorescence, which rely on photon absorption, SHG is a nonlinear scattering process; hence, in principle it deposits no energy to the matter with which the light interacts. Moreover, the SHG contrast can be endogenous, ${ }^{4}$ meaning that no additional staining is required. The energy conservation and label-free characteristics enable noninvasive imaging, which is highly desirable especially for in vivo studies. These features have increased the interest of SHG imaging for biomedical applications.

The most widely studied biological structures that produce an endogenous SHG signal are collagen, ${ }^{5-9}$ muscle, ${ }^{4,10-21}$ microtubules, ${ }^{4,2-24}$ starch, $^{25-27}$ and cellulose. ${ }^{25,28,29}$ The description of harmonic upconversion in the molecular arrangements of such structures can be attributed to coherently excited dipolar moments that generate hyper Rayleigh scattering ${ }^{30}$ analogous to phase array antennas. ${ }^{31,32}$ Constructive interference from an entire population of such structures (called harmonophores) results in the final harmonic signal. Furthermore, these elementary SHG active scatterers, when excited with different incoming linear polarizations (or equivalently by rotating the sample), provide a SHG response that is characteristic of the local scatterer geometrical arrange-

1083-3668/2009/14(1)/014001/11/\$25.00 ○ 2009 SPIE 
ment. This polarization-dependent SHG (PSHG) can be exploited as a new source of contrast in SHG imaging. PSHG data are usually analyzed using a theoretical model that assumes certain symmetry to the $\chi^{(2)}$ tensor. This technique was initially proposed to identify fiber orientation in collagen. ${ }^{7}$ In a similar way, PSHG in combination with the above analysis has been used to interpret images taken from muscles. ${ }^{14-19,21}$ By further assuming the single-axis molecule approach for the SHG active molecules, it was possible to calculate the mean effective orientation of the harmonophores and to probe myosin as the molecule responsible for the contrast. ${ }^{17-19}$ Recently, by inserting an analyzer before the signal detection, PSHG was reported to provide in situ selectivity between myosin and collagen. ${ }^{21}$ However, those results were obtained in myofibrils aligned along one predefined coordinate axis. Moreover, in those cases the PSHG intensity was averaged using large regions in the image (equal to the diameter of a single or several myofibrils). ${ }^{14-19,21}$

In this work we use a generalization of the PSHG technique $\mathrm{e}^{7-9,16}$ to study muscular nonfibrilar structures in a living organism, Caenorhabditis elegans (C. elegans). By using the generalized model, we show that it is possible to retrieve, at the pixel-resolution level, the ratios of the involved nonlinear susceptibility tensor elements, the angle of the thick filaments, and the distribution of the effective orientation of SHG active molecules. All the above are accomplished by using samples with no predefined muscle alignment, without any rotation of the sample, and without the use of analyzers before detecting the SHG signal. In addition, we also show that such a generalization can be used to retrieve multiple orientations of thick filaments in complex nonfibrilar muscle such as the ones contained in the pharynx of the nematode. In addition, we show that, after fitting the PSHG images to the model, the coefficient of determination $\left(r^{2}\right)$ can be used as a filtering mechanism to gain a new way of contrast. This method is able to uncover structures contained within the pharynx of the nematode that are not visible with conventional intensity SHG imaging.

This paper is organized as follows. First, we present the generalization of the biophysical model. We follow this with a discussion of its applicability and limitations. Then we apply the model to the well-organized thick filaments of the body walls, oriented along a predefined axis, to test our results at the pixel-resolution level. Next, we describe a second experiment in which the orientation of the thick filaments was set to an arbitrary angle to corroborate the generalized model. After this, we analyze single-sarcomere pharyngeal muscle cells in which thick filaments are nonuniformly oriented. Finally, the harmonophore orientation and experimental distribution is obtained.

\section{Materials and Methods}

\subsection{Caenorhabditis Elegans Muscles}

Although many studies have explored the basic functioning of muscles and their components, important aspects of sarcomere assembly and the contractile process are still not fully understood. C. elegans represents an attractive model system for microscopy studies ${ }^{33}$ on muscular function, because it presents large-sized sarcomeres. ${ }^{34}$ It also possesses the two types of muscle: striated and nonstriated. The obliquely striated body-wall muscles of this nematode consist of multiple sarcomeres, similar to the skeletal muscles in vertebrates. ${ }^{35}$ The nonstriated, single-sarcomere muscle cells are analogous to cardiac muscle cells in mammals and include the pharyngeal group. $^{35}$

The sarcomere consists of three differentiated regions. The isotropic (I) half-bands correspond to thin filaments made of actin; an anisotropic (A) band includes an overlapping region of thick filaments with thin filaments; the M-line, in the middle of the sarcomere, contains only thick filaments. The elements responsible for the SHG signal inside the sarcomere are the thick filaments. ${ }^{17}$ These are rigid structures based on a tubule-like core that constitutes the backbone of the filament. ${ }^{36-38}$ The internal density of the filament core appears hollow at the poles (similar to collagen type I fibrils), ${ }^{39}$ and they are made up of double-helix chiral myosin rods and heads. An interaction between the myosin heads and actin filaments produces muscle contraction and tension.

It has been reported that the SHG source in muscle lies in the rod domain of myosin's double helix, and that the myosin heads do not contribute to the signal. ${ }^{17}$ Furthermore, electron microscopy has shown that the spatial arrangement of thick filaments in the body walls, in the transverse direction, follows a hexagonal architecture. ${ }^{33}$ Based on this evidence, our model attributes hexagonal symmetry to the nonlinear susceptibility tensor in the sample.

\subsection{Worm Mounts}

The strain juIs76 [unc-25::gfp] II was cultured and grown in large quantities using methods reported by S. Brenner. ${ }^{40}$ The use of this specific strain relates to other research interests, but it is suitable for SHG muscular imaging because it only expresses green fluorescent protein (GFP) in a specific set of neurons. A number of healthy adult hermaphrodites were mounted on a $2 \%$ agar pad with $0.8 \mu \mathrm{l}$ of $25-\mathrm{mM}$ sodium azide (NaN3) between two $40-\mu \mathrm{m}$ glass slides. The choice of this specific anesthesia allowed us to fully immobilize the worms, and therefore reduce motion artifacts. The mounts were sealed with melted paraffin for stabilization and were only used for a period of less than half an hour to guarantee the physical condition of the worms. The laboratory temperature was $21^{\circ} \mathrm{C}$.

\subsection{Experimental Setup}

The experimental setup was based on an adapted inverted microscope (Nikon TE2000-U) with the $y$ - $z$ scanning unit composed of a pair of galvanometric (galvo) mirrors. A labVIEW interface program was written to control the raster scanning of the galvanometric mirrors and the data acquisition (DAQ) card. Typical frame acquisition times for a single $500 \times 500$ pixel image were about 1 to $2 \mathrm{~s}$. Two $60 \times$ oilimmersion objectives [numerical aperture $(\mathrm{NA})=1.4$, Nikon Plain Apo-Achromatic] were used throughout the experiments for excitation and collection of the nonlinear signals.

For the excitation source, we used a Kerr lens modelocked Ti:sapphire laser (MIRA 900f), which delivers pulses of $160 \mathrm{fs}$ and operates at a central wavelength of $840 \mathrm{~nm}$ with a repetition rate of $76 \mathrm{MHz}$. The average power reaching the sample plane was controlled (using neutral density filters) to be in the range of 20 to $30 \mathrm{~mW}$. With this regime, no 
observable damage occurred for long imaging periods of time. We placed a linear polarizer after the galvo mirrors to eliminate any ellipticity or depolarization introduced from the setup. This was followed by a half-wave plate that was rotated in steps to change the polarization at the sample plane. For the analysis, we defined the lab $z$ axis in the horizontal direction in our images. This corresponded to the angle origin $(\alpha=0)$ of the incoming linear polarization. Subsequent linear polarizations were used by rotating them clockwise in steps of $20 \mathrm{deg}$. Finally, we assessed the effect of several optical components in our microscope placed before the sample plane (galvo mirrors, lenses, half-wave plate, dichroic mirror, and objective) on the depolarization of the fundamental beam. This was done by measuring the power of the fundamental beam as a function of the rotation angle of an analyzer placed at the microscope sample plane and before the power meter. We found that the fundamental input average power showed an extinction coefficient ratio of 25:1 under rotation of the input polarization.

Since the great majority of the SHG signal generated from muscular tissues is forward propagated, a proper mount and detection unit was implemented in this direction. This unit contained the collecting high numerical aperture $(\mathrm{NA}=1.4)$ objective, a BG39 filter, a 10-nm FWHM band-pass filter centered at $420 \mathrm{~nm}$ and a photomultiplier tube (PMT). The objective lens was mounted on a micrometric 3-D translational stage with tilt correction, and the whole unit was enclosed to minimize stray or spurious light into the PMT.

\subsection{PSHG Biophysical Model}

Our generalized biophysical model is based on those proposed previously. ${ }^{14-18}$ For clarity, we describe it here while introducing the generalization, which allows the acquisition of any arbitrary orientation $\phi$ of the thick filaments.

Our model first considers that the SHG active volume in our experiment $\left(\sim 0.1 \mu \mathrm{m}^{2} \times 0.8 \mu \mathrm{m}\right)$ is smaller than the size of a sarcomere, but it includes several thick filaments (considered the source of SHG signal in muscle ${ }^{17}$ ) that are hexagonally arranged. ${ }^{33}$ Therefore, a spatial symmetry corresponding to hexagonal crystal system is assumed. Second, we further consider that the thick filaments are at an arbitrary angle orientation and parallel to the sample plane of the microscope (see Fig. 1).

To be formally correct, we assume that the local hexagonal arrangement of thick filaments was transduced in a $C_{6}$ crystal class whose susceptibility tensor in its contracted notation is given by ${ }^{41}$

$$
\bar{d}=\left[\begin{array}{cccccc}
0 & 0 & 0 & d_{14} & d_{15} & 0 \\
0 & 0 & 0 & d_{15} & -d_{14} & 0 \\
d_{31} & d_{31} & d_{33} & 0 & 0 & 0
\end{array}\right],
$$

where $d_{i^{\prime} j^{\prime}}=\chi_{i^{\prime} j^{\prime}} / 2$. The coordinate frame associated with the thick filaments $\left(x^{\prime}, y^{\prime}, z^{\prime}\right)$ is chosen with $z^{\prime}$ along the direction of the thick filaments (see Fig. 1). Then the components of the nonlinear polarization response can be expressed in terms of an arbitrary incident fundamental electric field $\left(E_{x^{\prime}}^{\omega}, E_{y^{\prime}}^{\omega}, E_{z^{\prime}}^{\omega}\right)$ as

$$
P_{x^{\prime}}^{2 \omega}=2 \chi_{14}^{(2)} E_{y^{\prime}}^{\omega} E_{z^{\prime}}^{\omega}+2 \chi_{15}^{(2)} E_{x^{\prime}}^{\omega} E_{z^{\prime}}^{\omega},
$$

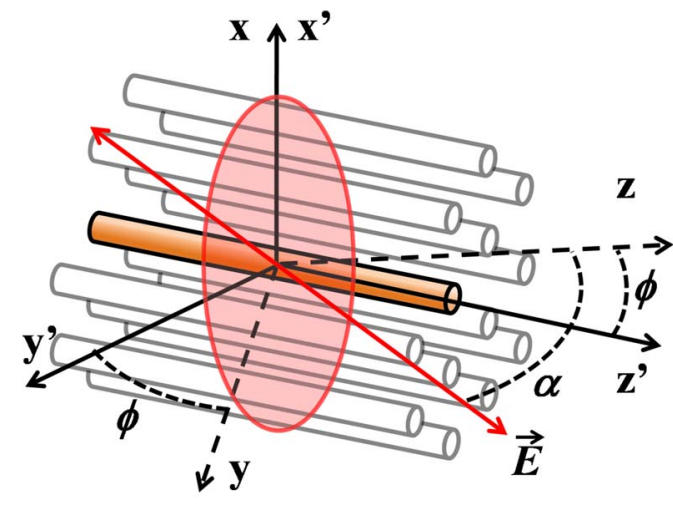

Fig. 1 Schematic representation of coordinate system inside the focusing volume. The beam propagates along the $x$ direction, and the main symmetry axis is in an angle $\phi$ with the $z$ axis. $\alpha$ is the angle of the incoming linear polarization with the $z$ axis. Cylinders represent thick filaments, and the shadowed region corresponds to the SHG active volume. Figure is not on a predefined scale.

$$
\begin{gathered}
P_{y^{\prime}}^{2 \omega}=-2 \chi_{14}^{(2)} E_{x^{\prime}}^{\omega} E_{z^{\prime}}^{\omega}+2 \chi_{15}^{(2)} E_{y^{\prime}}^{\omega} E_{z^{\prime}}^{\omega}, \\
P_{z^{\prime}}^{2 \omega}=\chi_{31}^{(2)}\left(E_{x^{\prime}}^{\omega}\right)^{2}+\chi_{31}^{(2)}\left(E_{y^{\prime}}^{\omega}\right)^{2}+\chi_{33}^{(2)}\left(E_{z^{\prime}}^{\omega}\right)^{2} .
\end{gathered}
$$

This results in a radiated SHG electric field given by

$$
\vec{E}^{2 \omega} \propto \hat{k} \wedge\left(\vec{P}^{2 \omega} \wedge \hat{k}\right)
$$

where $\hat{k}$ is the SHG unitary wave vector. The contribution of each term in Eqs. (2)-(4) to the radiated SHG field given by Eq. (5) depends on the actual experimental conditions. In this work, the thick filaments are assumed to be parallel to the microscope sample plane, i.e., in the $y-z$ plane, while the laser beam propagates in the $x$ direction. With this geometry, the $x$ (lab) and the $x^{\prime}$ (thick filament) axes coincide, and the relation between the microscopic (filaments) and macroscopic (laboratory) frames corresponds to a rotation characterized by an angle $\phi$ (note that this angle is the thick filament orientation with respect to the lab frame). In addition, to obtain a close expression for the collected SHG signal, we restrict such a SHG-radiated signal to be unidirectional, with $\hat{k}=\hat{x}$. Since we consider that $\hat{k}$ is parallel to the optical axis of the microscope, then there is no contribution of the nonlinear polarization component $P_{x^{\prime}}^{2 \omega}$.

We further consider a linear polarization of the incident electric field in which no axial component $E_{x^{\prime}}^{\omega}$ is introduced by our high-NA $(\mathrm{NA}=1.4)$ objective. In our experiment the polarization is rotated clockwise with an angle $\alpha$ measured with respect to the $z$ axis (lab macroscopic frame). The amplitude of the electric field at the focal position, expressed in the thick filaments coordinate frame, is given by

$$
\vec{E}^{\omega}=E_{0}\left[\sin (\phi-\alpha) \hat{y}^{\prime}+\cos (\phi-\alpha) \hat{z}^{\prime}\right] .
$$

Then, by introducing Eq. (6) into Eqs. (2)-(4) and using Eq. (5), the SHG field can be written as 


$$
\begin{aligned}
\vec{E}^{2 \omega} \propto & E_{0}^{2}\left\{2 \chi_{15}^{(2)} \sin (\phi-\alpha) \cos (\phi-\alpha) \hat{y}^{\prime}+\left[\chi_{31}^{(2)} \sin ^{2}(\phi-\alpha)\right.\right. \\
& \left.\left.+\chi_{33}^{(2)} \cos ^{2}(\phi-\alpha)\right] \hat{z}^{\prime}\right\} .
\end{aligned}
$$

Since in this study no analyzer is used in the detection path, the total SHG intensity can be obtained directly [without transforming Eq. (7) to the lab frame]:

$$
\begin{aligned}
I^{2 \omega} \propto & I_{0}\left(\chi_{15}^{(2)}\right)^{2}\left\{\sin ^{2}(\phi-\alpha) \cos ^{2}(\phi-\alpha)+\left[\frac{\chi_{31}^{(2)}}{\chi_{15}^{(2)}} \sin ^{2}(\phi-\alpha)\right.\right. \\
& \left.\left.+\frac{\chi_{33}^{(2)}}{\chi_{15}^{(2)}} \cos ^{2}(\phi-\alpha)\right]^{2}\right\} .
\end{aligned}
$$

This equation includes information on both the tensor elements and the thick filament orientation. It has to be noted that equation (8) has been obtained only by assuming hexagonal symmetry (based on electron microscopy evidence ${ }^{33}$ ) defined in a coordinate system without the need of extra conditions such as Keinman's symmetry condition. However, due to the simplifications used, the hexagonal symmetry of the structure is in the end reduced into a cylindrical symmetry of nonlinear dipoles around the filament axis.

\subsection{Single-Axis Molecule Approach}

By expanding the model to the microscopic (molecular) frame and by considering a coherent summation of the molecular hyperpolarizability tensor $\beta_{i^{\prime \prime} j^{\prime \prime} k^{\prime \prime}}$, the different elements in the macroscopic nonlinear susceptibility tensor can be obtained. In addition, it is generally assumed that the single-axis molecules posses a unique nonvanishing element $\beta_{z^{\prime \prime} z^{\prime \prime} z^{\prime \prime}}=\beta .^{17,18,42}$ Then the components of the susceptibility tensors are obtained:

$$
\chi_{i^{\prime} j^{\prime} k^{\prime}}^{(2)}=\sum_{i^{\prime \prime} j^{\prime \prime} k^{\prime \prime}}\left\langle\cos \theta_{i^{\prime} z^{\prime \prime}} \cos \theta_{j^{\prime} z^{\prime \prime}} \cos \theta_{k^{\prime} z^{\prime \prime}}\right\rangle \beta .
$$

Using the angles $\theta$ and $\varphi$ in spherical coordinates, the different tensor elements can be expressed as

$$
\begin{gathered}
\chi_{33}^{(2)}=\beta N\left\langle\cos ^{3} \theta\right\rangle, \\
\chi_{31}^{(2)}=\chi_{15}^{(2)}=\beta N\left\langle\cos \theta \sin ^{2} \theta \sin ^{2} \varphi\right\rangle, \\
\chi_{14}^{(2)}=\beta N\left\langle\sin ^{2} \theta \cos \theta \sin \varphi \cos \varphi\right\rangle,
\end{gathered}
$$

where $N$ is the density of molecules, and \langle\rangle denotes an orientation average over the distribution of molecular orientations $D(\theta, \varphi)$. When the assumption of single-axis molecules is applied in Eq. (9), it results in $\chi_{31}^{(2)} / \chi_{15}^{(2)}=1$ in Eq. (11). An experimental deviation of this ratio from the unit can be attributed to either experimental errors or to a deviation from the single-axis molecules approach.

By considering a molecular random distribution in the azimuth angle $(\varphi)$, then $D(\theta, \varphi)=D(\theta)$, and Eq. (11) reduces to $\chi_{31}^{(2)}=\chi_{15}^{(2)}=\beta N\left\langle\cos \theta \sin ^{2} \theta\right\rangle / 2$. Equivalently, Eq. (12) would result in $\chi_{14}^{(2)}=0$. However, this aspect cannot be corroborated since our model is not able to retrieve information on this tensor element.
Finally, assuming a square distribution for $D(\theta)$, the mean effective molecule or harmonophore orientation can be estimated as ${ }^{18,42}$

$$
\cos ^{2} \theta_{e}=\frac{\chi_{33}^{(2)} / \chi_{15}^{(2)}}{2+\chi_{33}^{(2)} / \chi_{15}^{(2)}}
$$

Alternatively, Eq. (13) can also be obtained by attributing a Dirac delta distribution to the molecule orientation, i.e., $D(\theta)=\delta\left(\theta-\theta_{e}\right)$. Then the meaning of Eq. (13) would correspond to the actual orientation in the analyzed region. The suitability of the two approaches is discussed in Sec. 3.

\subsection{Fitting Method}

In order to make apparent the fitting process, Eq. (8) is rewritten as

$I^{2 \omega} \propto I_{f}\left\{\sin ^{2} 2(\phi-\alpha)+\left[a \sin ^{2}(\phi-\alpha)+b \cos ^{2}(\phi-\alpha)\right]^{2}\right\}+\Delta$.

using Eq. (14), information on the thick filament orientation and nonlinear susceptibility components can be extracted. In this case, the free parameters $I_{f}, a, b, \phi$, and $\Delta$ are retrieved using a fitting algorithm. The number of required polarization angles $\alpha$ can be estimated by making use of the Nyquist theorem (the sampling rate must be twice the higher frequency component). Since the higher frequency in Eq. (14) is $4 / 2 \pi \mathrm{rad}^{-1}$ (taking into account the power 4 ), then the resulting polarization sampling should be every $8 / 2 \pi \mathrm{rad}^{-1}$ (45 deg). Equation (14) has a period of $180 \mathrm{deg}$, so the minimum number of polarization angles would be 4 . However, we prefer to use nine measurements at different polarizations and a fitting algorithm to retrieve the free parameters, which minimizes the impact of experimental errors by increasing the information. This was based on a nonlinear least-squares fitting routine (The Mathworks, Champaign-Urbana, IL).

The difference between Eqs. (8) and (14) is in the extra term $\Delta$. This parameter has been added to include both experimental errors and any deviation from the theoretical model, so it is not to be considered a simple background contribution. This is because the minimum value in Eq. (14), without considering any background, can be larger than zero depending on the actual values of $a$ and $b$. This makes it difficult to a priori quantify the contribution of $\Delta$ to the detected signal. In addition, $\Delta$ can slightly change from pixel to pixel. Therefore, it has been included as a free parameter in the fitting algorithm.

Our fitting algorithm produces an intrinsic indetermination. This occurs because when $a=\chi_{31}^{(2)} / \chi_{15}^{(2)}$ and $b=\chi_{33}^{(2)} / \chi_{15}^{(2)}$, it gives the same result at $\phi$ as $b=\chi_{31}^{(2)} / \chi_{15}^{(2)}$ and $a=\chi_{33}^{(2)} / \chi_{15}^{(2)}$ at $\phi+\pi / 2$. To solve this, we imposed the condition that the larger of the two coefficients $a$ or $b$ given by the fitting algorithm must correspond to $\chi_{31}^{(2)} / \chi_{15}^{(2)}$, and the remaining coefficient and angle must be chosen accordingly. This assumption is correct only in the case of muscle ${ }^{18}$ and therefore must be revised for other SHG active structures. In addition, since all the terms in Eq. (8) are positive, the algorithm is forced to retrieve positive real values for all the parameters in Eq. (14). Thus, spurious solutions, usually with negative values for either $a$ or $b$ and $\Delta$, are avoided. 
Having implemented all these observations, we applied our fitting algorithm to each pixel of a selected region of interest (ROI). This was done after applying a low-pass filter (equivalent to averaging the four surrounding neighboring pixels) to remove any possible small-moving artifacts. The average intensity among five different images of the same plane and polarization was used to obtain an accurate analysis. Under these conditions, over 1000 iterations per pixel were used to adjust the experimental data to the model. The free image processing software Image $\mathbf{J}$ (National Institutes of Health) was used for image treatment.

\section{Results and Discussion}

\subsection{Considerations of the Theoretical Model}

Three important considerations have not yet been considered by our model: (1) the SHG signal reaching the collecting objective with off-axis $\hat{k}$ vectors (within the NA); (2) the change of the polarization state (existence of axial components) due to the high-NA objective, and (3) the effect of thick filaments tilted off-plane. The consideration of off-axis $\hat{k}$ vectors and a close equation for the intensity, ${ }^{43}$ are making the analysis numerically complex The two remaining aspects, axial field components and off-plane filaments, could be included in the model by adding an extra $\psi$ angle to complete the Euler set of angles and by using analyzers in detection to determine this angle. ${ }^{42}$ All this would also incorporate the $\chi_{14}^{(2)}$ tensor element in our model, whose inclusion would add complexity to the model by introducing extra ambiguities into the equation.

In view of the above discussion, we decided to keep our simpler approach and instead to analyze how these three factors could affect our results. In all three cases, the main effect is expected to be an extra contribution of the nonlinear polarization component $P_{x^{\prime}}^{2 \omega}$ to the detected SHG signal (although $P_{y^{\prime}}^{2 \omega}$ and $P_{z^{\prime}}^{2 \omega}$ can also change). In principle, this SHG signal due to $P_{x^{\prime}}^{2 \omega}$ would drop very fast (nonlinearly) and could be $a$ priori disregarded. However, our fitting algorithm has the potential to consider its contribution and to minimize its impact on the retrieved results thanks to the inclusion of the $\Delta$ parameter.

The experimental background (electronic noise, nonfiltered fundamental signal, or external light) can be estimated by measuring the intensity in regions without any SHG signal. This corresponds to $\sim 1 \%$ of the detected signal. However, the value of the $\Delta$ parameter is larger and can change from pixel to pixel. Typically, in an image, $\Delta$ is around $20 \%$ of the detected signal with a change of 2 to $3 \%$ from pixel to pixel. Its nature can be associated with the causes mentioned above, as well as the existence of other factors such as two-photon fluorescence. A certain dependence of $\Delta$ with the incoming polarization is expected. However, when determining $\Delta$, the fitting algorithm does not take into account this dependence, so it gives an average value. As a consequence, the fitting algorithm removes part of the unwanted contributions included in $\Delta$. This is shown in Figs. 3(b), 4(b), and 5(b), where the quality of fitting for most of the pixels was above $85 \%$; when $\Delta$ was not included, the quality of fitting was always below $70 \%$.

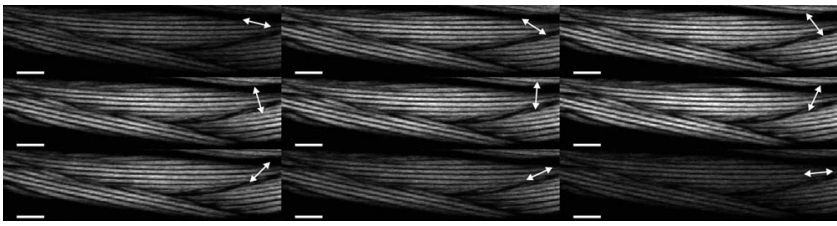

Fig. 2 Polarization-dependent SHG images of $C$. elegans ventral quadrants of body wall muscles. Incoming linear polarization is indicated by arrows and is rotating in steps of $20 \mathrm{deg}$. Scale bar shows $10 \mu \mathrm{m}$.

A final remark on the method concerns Kleinman's symmetry conditions. In our experiments, these conditions imply that $\chi_{14}^{(2)}=0$ and $\chi_{31}^{(2)}=\chi_{15}^{(2)}$. The determination of the $\chi_{31}^{(2)} / \chi_{15}^{(2)}$ ratio can give a measure of the validity of those conditions. In our case, Figs. 3(c), 4(c), and 5(c) show that a large number of pixels returned an $a$-parameter value that was different from unity. This is an unexpected result if Kleinman's conditions hold. To further check this fact, the fitting algorithm was run by assuming Kleinman's symmetry conditions, i.e., $\chi_{31}^{(2)} / \chi_{15}^{(2)}$ $=1$. The results showed a much lower quality of fitting (below $60 \%$ ). In addition, the SHG signal at $\lambda=420 \mathrm{~nm}$ was very close to the resonant wavelength of the muscle at $\lambda$ $\sim 430 \mathrm{~nm} .{ }^{44}$ Thus, care must be taken before assuming Kleinman's symmetry conditions in experiments of this type.

\subsection{Caenorhabditis Elegans Body Walls}

In vivo, PSHG imaging of $C$. elegans body wall muscles for nine different incoming polarizations are shown in Fig. 2. Each image presented in this figure was composed by the mean intensity of five different images of the same polarization. As expected, there was clear variation of the intensity among the different SHG images. In this set of experiments, we found that the minimum PSHG intensity was observed when the incoming polarization was almost parallel with the thick filament orientation. Our results showed that the distinctive intensity PSHG fingerprint curve characteristic of muscle (result not shown) were in agreement to previous results in which myofibrils were oriented parallel to one axis of the coordinate system. ${ }^{14-19,21}$

In this section, we analyze the images using pixel resolution. This is in contrast to previous PSHG studies on muscle where the results were obtained by averaging the intensity from large ROIs. ${ }^{14-19,21}$ Averaging over bigger regions would give smoother changes among any retrieved values.

We start by using the results in Fig. 2 to run our pixel resolution fitting algorithm to the selected ROI (where the thick filaments are mainly parallel to the $z$ axis), shown in Fig. 3(a). Figure 3(b) shows the pixels that were fitted with a coefficient of determination of $r^{2} \geqslant 95 \%$ inside the selected ROI. Figures 3(c)-3(f) show the $a$ and $b$ parameters, the angle of thick filaments, and the effective orientation of harmonophores, respectively. In these figures, large homogenous regions are shown (indicated with the same color) for the different parameters of the model. As commented previously, Fig. 3(c) exhibits some deviation from the situation $\chi_{31}^{(2)} / \chi_{15}^{(2)}=1$ necessary for Kleinman's symmetry to hold, with the majority of the pixels being around 1.4. For the structurally sensitive coefficient $\chi_{33}^{(2)} / \chi_{15}^{(2)}$, the ratio obtained 


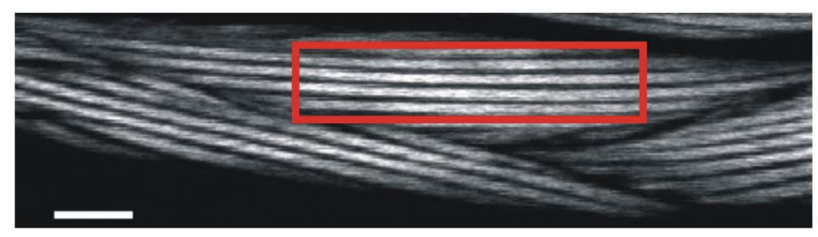

(a)

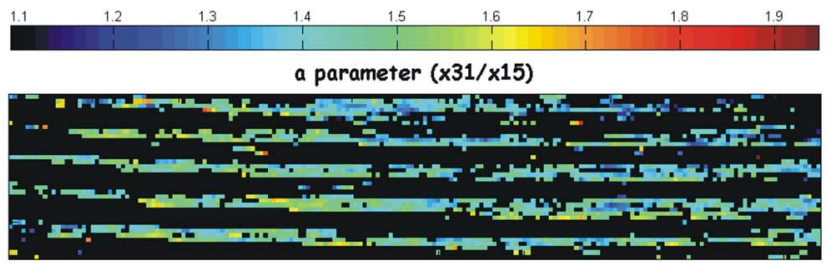

(c)

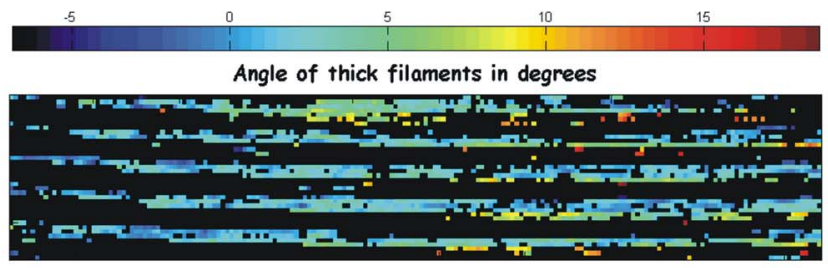

(e)

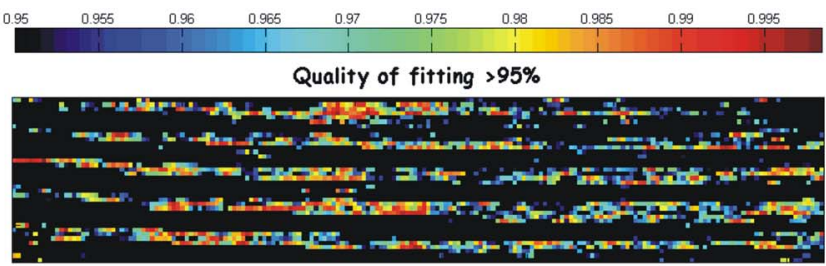

(b)

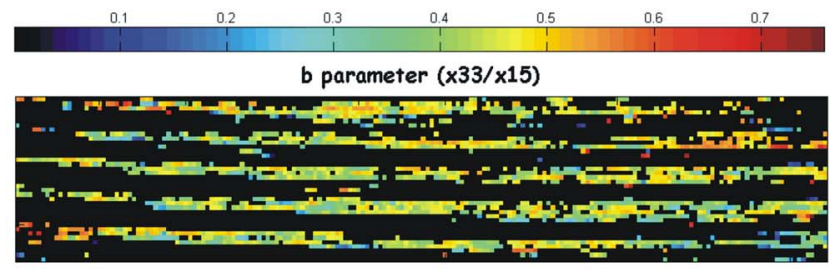

(d)

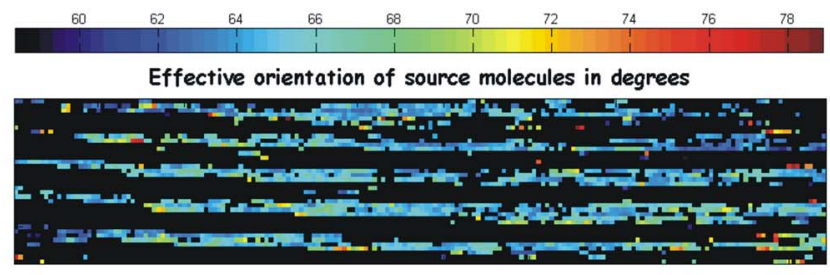

(f)

Fig. 3 Algorithm fitting results: (a) Average intensity of all the SHG images shown in Fig. 2. The red square shows the ROI where the fitting algorithm was applied. Scale bar corresponds to $10 \mu \mathrm{m}$. (b) Quality of fitting $\left(r^{2}\right)$. (c) and (d) Obtained ratios between the elements of the $\chi^{(2)}$ tensor. (e) Orientation of thick filaments $(\phi)$. (f) Effective orientation of harmonophores $\left(\theta_{e}\right)$, both in degrees. For clarity, the values with a quality of fitting $\left(r^{2}\right)$ inferior to $95 \%$ were rejected from the figures. (Color online only.)

for the majority of the pixels was at 0.45 [Fig. 3(d)]. These values are in agreement with those previously reported for myofibrils. ${ }^{14-19}$ Figure 3(e) clearly shows that the retrieved angle $\phi$ of thick filaments coincides well with the geometrical inclination observed in the ROI of Fig. 3(a) (angles between 0 and $5 \mathrm{deg}$ ). Figure 3(f) shows the effective orientation angle of the harmonophores calculated using Eq. (13). The maximum number of pixels presented an angle in the region of $64.1 \mathrm{deg}$. These results will be discussed further later.

In the next step, the $C$. elegans body wall muscles were examined at an arbitrary inclination with respect to the $z$ axis [see Fig. 4(a)]. In this way, it was possible to test if the generalization of the model including the angle $\phi$ was valid. To do that, we followed the same procedure as before. It was found that the PSHG fingerprint curve $\mathrm{e}^{14-19,21}$ was shifted according to the angle $\phi$. The results of the fitting for $r^{2}$ $>85 \%$ are depicted in Figs. 4(b)-4(f). The values of the different parameters are at the same intervals as those acquired before (body walls parallel to the $z$ axis). The maximum number of pixels for the $a, b$, and $\theta_{e}$ parameters was in the regions of $1.03,0.38$, and $65.5 \mathrm{deg}$, respectively. In the case of the angle $\phi$ [Fig. 4(e)], the majority of the pixels showed an inclination for the thick filaments of $58.47 \mathrm{deg}$ - very close to the real geometrical inclination in the SHG image. This clearly indicates that the method can correctly assess the orientation of thick filaments, and therefore, the sample does not need special alignment before starting the measurements. This advantage is crucial, especially when examining complex architectures, since it allows the simultaneous pixel resolution mapping of different orientations in the same image.

\subsection{Complex Thick Filament Architectures: The Terminal Lobe of the Caenorhabditis Elegans Pharynx}

Although the orientation of the thick filaments in body walls could be easily predicted, in more complex structures such as the pharynx, the analysis should provide information not apparent from conventional SHG intensity imaging. Thus, our next objective was to analyze complex muscular structures that possess different orientations of the thick filaments in the same image.

The pharynx of $C$. elegans was used to approach this issue. The pharynx is a single, unified, epithelial bilobed organ composed of 20 cells, and it can be divided into eight distinct muscle groups, each containing one to three cells. Within the pharynx we selected a $y-z$ plane of a region in the most posterior lobe (the terminal bulb). This specific region of the pharynx was selected because it includes cells from three different muscle groups (pm5, pm6, and pm7) with different filament orientations. Moreover, the posterior lobe of the $C$. elegans pharynx has a highly complex organized structure where the muscular cells are organized on a threefold radial symmetry, with marginal cells in between, that rotate under contraction and allow for both a longitudinal and radial component to the motion of the grinder teeth (housed in the posterior lobe) during food intake. ${ }^{33}$

To test the ability of our model to map the different orientations of the thick filaments in the same image, we performed 


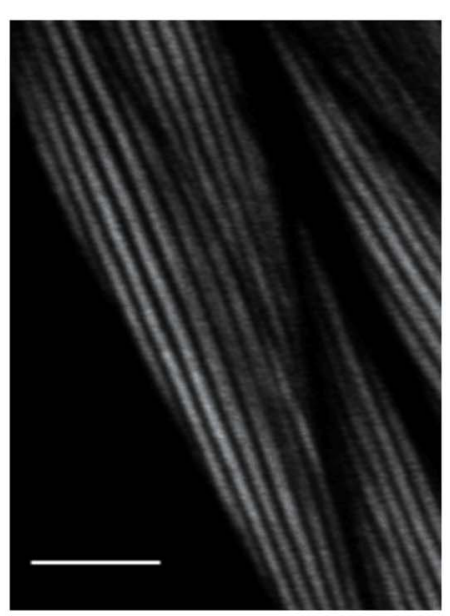

(a)

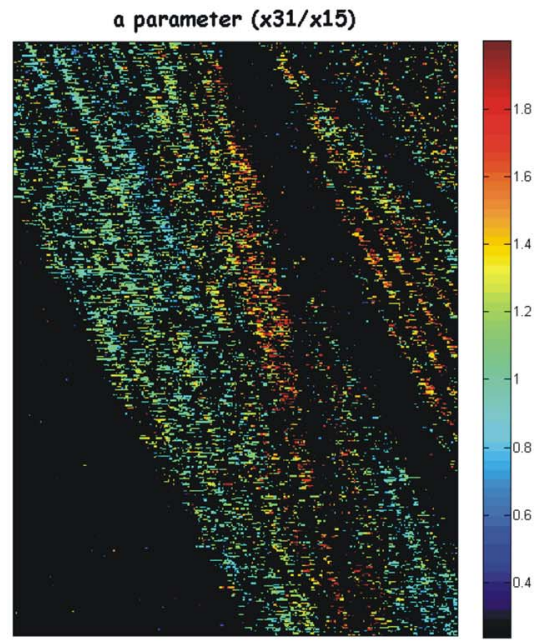

(c)

Angle of thick filaments in degrees

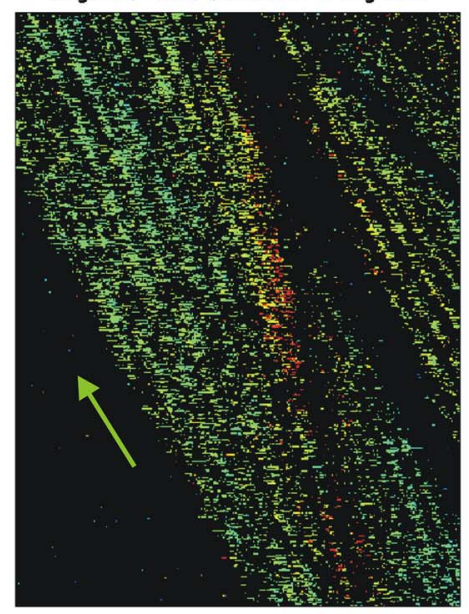

(e)

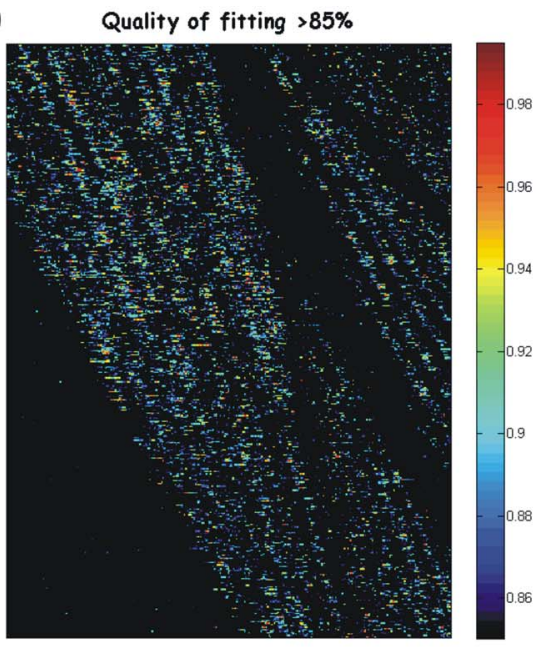

(b)

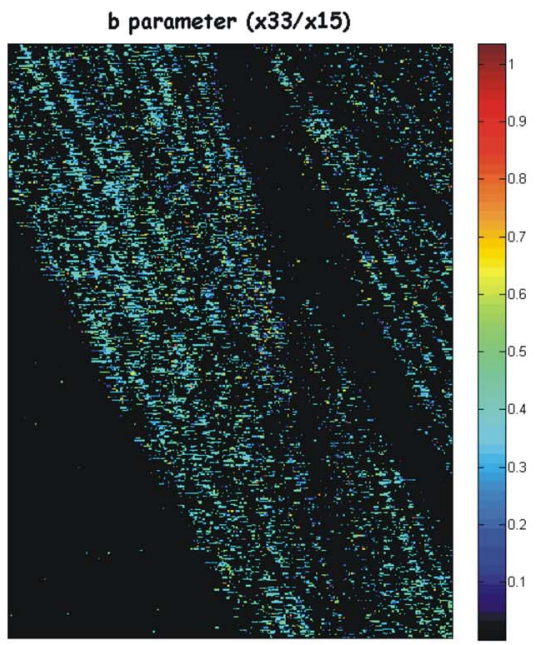

(d)

Effective orientation of source molecules in degrees

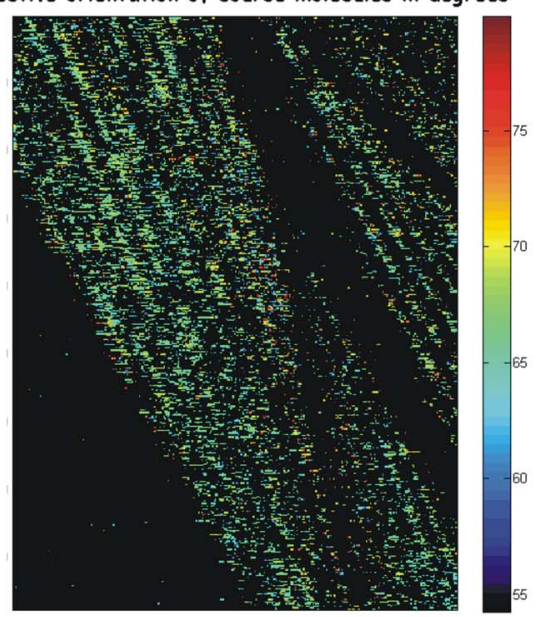

(f)

Fig. 4 Results of fitting the algorithm to body walls oriented at an arbitrary inclination angle. (a) Average intensity of nine different PSHG images (each step in the incoming linear polarization is $20 \mathrm{deg}$ ). Scale bar is $10 \mu \mathrm{m}$. (b) Coefficient of determination $\left(r^{2}\right)$. (c) $a=\chi_{31}^{(2)} / \chi_{15}^{(2)}$ and (d) $b$ $=\chi_{33}^{(2)} / \chi_{15}^{(2)}$ are obtained ratios between the elements of the $\chi^{(2)}$ tensor. (e) Orientation of thick filaments $(\phi)$. Arrow indicates real inclination at 58.47 deg. (f) Effective orientation of harmonophores $\left(\theta_{e}\right)$ in degrees. 
the PSHG image analysis to the pharynx (see Fig. 5). The results of the fitting for the several structurally sensitive parameters of the model can be seen in Figs. 5(c)-5(f). In these figures, only values of $r^{2}>90 \%$ are presented [Fig. 5(b)]. The maximum number of pixels for the $a, b$, and $\theta_{e}$ parameters was found at the values of $0.94,0.52$, and $63.02 \mathrm{deg}$, respectively. Figure 5(e) shows the obtained values for the angle $\phi$, which clearly demonstrates the radial distribution of thick filaments present in the most peripheral regions of both pm6 and pm7. Also in this figure, the pixel-by-pixel fitting of the algorithm resulted in a number of homogenous regions (shown with the same color or totally black). These regions are indicative of different angle orientations (different colors) or different structural characteristics (black) that did not fit to the algorithm. In all these cases, the different homogeneous regions can be distinguished from nearby regions. In the case of differently color regions, the obtained angle orientation hints at the local contraction direction of the sarcomere. Moreover, the fact that it is possible to choose coefficients of determination larger than a predefined value offers a new way of gaining contrast, because it filters PSHG pixels that do not fit to the model. Here, this fast was used to segment among different architectures. For example, using $r^{2}>90 \%$ allowed us to uncover the several parts of muscular cells that control this nematode's grinder in the pumping system (white arrow in Fig. 5). This information could not be reached with $r^{2}$ $<90 \%$ (data not shown) or by our conventional intensity SHG images [see Fig. 5(a)]. Also, the above result would not have been achieved without the generalization of the model (which allows mapping of different orientations of thick filaments in the same image) and without applying it at the pixelby-pixel level.

\subsection{Effective Orientation of SHG Active Molecules}

Since the effective orientation of the harmonophores [see Eq. (13) and Figs. 3(f), 4(f), and 5(f)] is measured with respect to the thick filament, such angles have been associated with the helical pitch of myosin. ${ }^{17,18}$ Such an association was made only after analyzing images of homogeneous striated fibrilar muscle samples in large ROIs and by assuming a square distribution of molecular orientations. In contrast, our pixelresolution procedure allows for nonhomogeneous regions to be analyzed as the different structural parameters are obtained in a pixel-by-pixel basis. Therefore, the statistical molecular distribution $D(\theta)$ in a single pixel is expected to be narrower. As a consequence, it is more appropriate to consider the local distribution in Eqs. (10)-(12) as a Dirac delta. As previously mentioned, the effective angle $\theta_{e}$ given by Eq. (13) is then expected to be closer to the actual harmonophore orientation in the pixel. This further allows us to experimentally estimate the actual distribution of effective molecular orientations in the whole image. Thus, we calculated the frequency distribution of the angles given by Eq. (13) for our data [Figs. 3(f), $4(f)$, and 5(f)]. These results, together with the frequency distribution corresponding to parameters $a=\chi_{31}^{(2)} / \chi_{15}^{(2)}$ and $b$ $=\chi_{33}^{(2)} / \chi_{15}^{(2)}$, are shown in Fig. 6 .

The frequency distribution for the ratio $\chi_{31}^{(2)} / \chi_{15}^{(2)}$ shown in Fig. 6(a) exhibits a "well-behaved" bell-shaped distribution in all cases. The FWHM is similar in the horizontal body walls and the body walls in an angle, but broader for the pharynx (even if a smaller ROI is considered). This broadening also occurs in the frequency distribution of the ratio $\chi_{33}^{(2)} / \chi_{15}^{(2)}[$ Fig. $6(b)]$ and as expected, in the frequency distribution of the effective orientation of harmonophores [Fig. 6(c)]. In this last case, the FWHM of such distribution, $\delta \theta_{e}$, is indicative of the degree of disorganization of the SHG source molecules. However, $\delta \theta_{e}$ for the horizontal body walls and for those in an angle were found to be similar, $\sim 5 \mathrm{deg}$, while for the pharynx $\delta \theta_{e}$ was $\sim 15 \mathrm{deg}$. These $\delta \theta_{e}$ were narrow enough to allow the accurate determination of the mean $\theta_{e}$ found in other works. ${ }^{45}$ The nature of the broader $\delta \theta_{e}$ in the pharynx might be attributed not only to the fact that thick filaments are not well organized, but also because some of these thick filaments may also tilted off of the sample plane.

The single-axis-molecules approach also imposes a ratio of $\chi_{31}^{(2)} / \chi_{15}^{(2)}=1$. As shown in Fig. 6(a), the body walls in an angle [Fig. 4(c)] and the pharynx lobe [Fig. 5(c)] are centered at $\chi_{31}^{(2)} / \chi_{15}^{(2)}=1$. This is not the case for the horizontal body walls, where a value centered at $\chi_{31}^{(2)} / \chi_{15}^{(2)} \approx 1.4$ was obtained. Although the reason for this is not clear, we note that the retrieved distribution for the ratio $\chi_{33}^{(2)} / \chi_{15}^{(2)}$ and the effective angle distribution is similarly centered in all three cases. This makes us believe that the divergence from the expected value $\chi_{31}^{(2)} / \chi_{15}^{(2)}=1$ might be due to experimental errors rather than to a lack of validity of the single-axis-molecules approach. In view of these results, we can conclude that the experimental condition that better matches our theoretical model corresponds to body walls in an angle (Fig. 4). In this case, the FWHM harmonophore orientation distribution falls in the range 63 to $67 \mathrm{deg}$ with a maximum at $\theta=65.5 \mathrm{deg}$. This value is, as previously mentioned, ${ }^{17,18}$ close to the myosin helical pith measured using x-ray diffraction, which is $68.6 \mathrm{deg}$, corroborating the validity of our pixel-resolution results.

\section{Conclusions}

In this study we used a generalization of a biophysical model that allows PSHG microscopy to retrieve the orientation of the thick filaments in nonfibrilar muscles of a living $C$. elegans. This generalization made the method free of any predefined specimen alignment, and consequently, it provided the means for mapping multiple different orientations of the thick filaments in the same image at the pixel-by-pixel level. By fitting the PSHG images to the model, we also obtained the local value and frequency distribution for the ratios between elements of the $\chi^{(2)}$ tensor and the effective orientation of the active SHG molecules (harmonophores). In the latter case, the obtained values coincided with those of the helical pitch of myosin, showing the validity of the method.

We examined well-ordered filaments of the body walls of the nematode oriented parallel and at an inclination angle from a predefined axis of a coordinates system. In both cases, the results of the fitting demonstrated that the correct information can be retrieved with pixel resolution. Using this background, we analyzed a region of the complex muscle groups of cells from the terminal lobe of the pharynx of $C$. elegans. In this case the pixel-resolution fitting of the model enabled us to identify the different orientations and to expose the radial distribution of the thick filaments. As a result, the specialized 


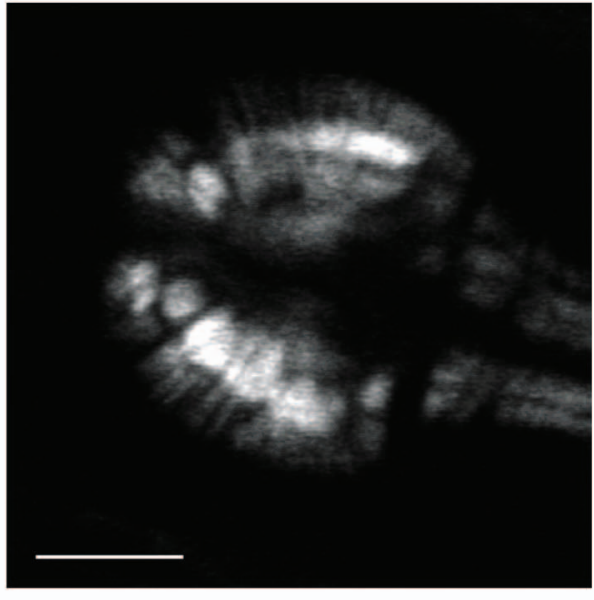

(a)

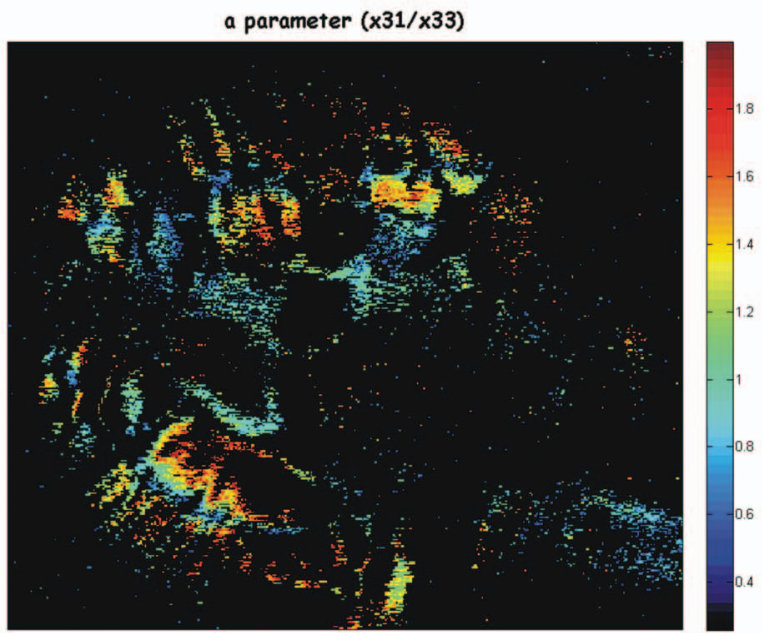

(c)

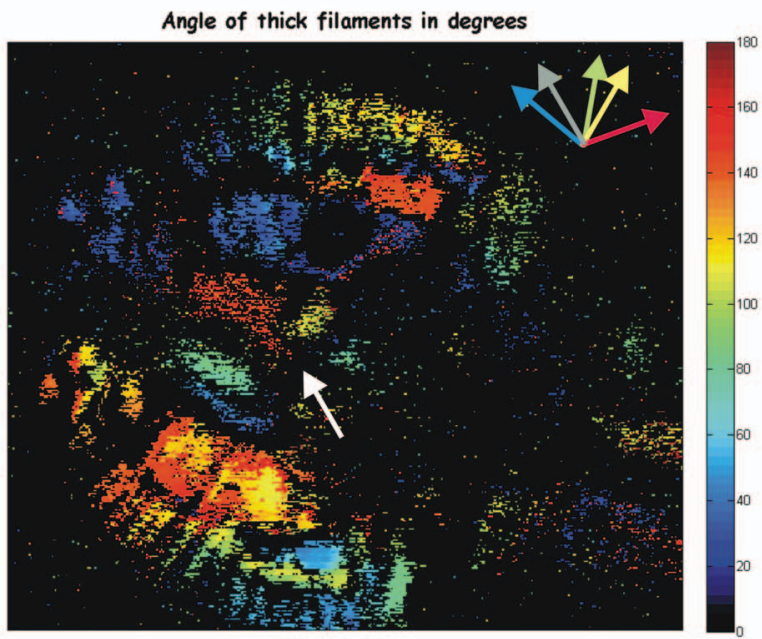

(e)

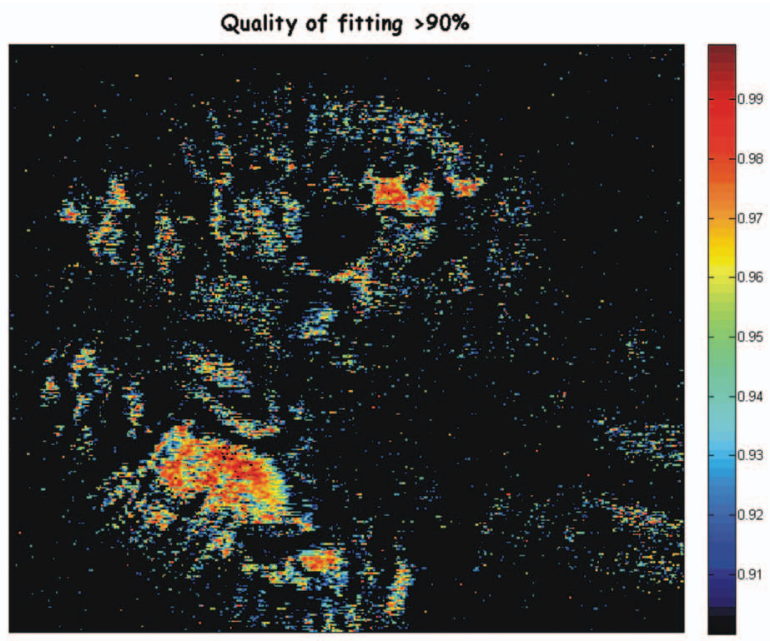

(b)

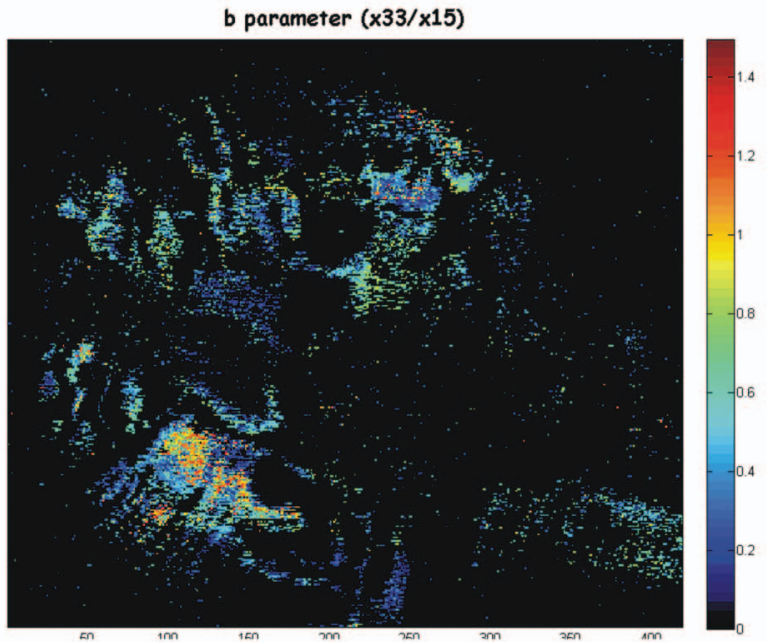

(d)

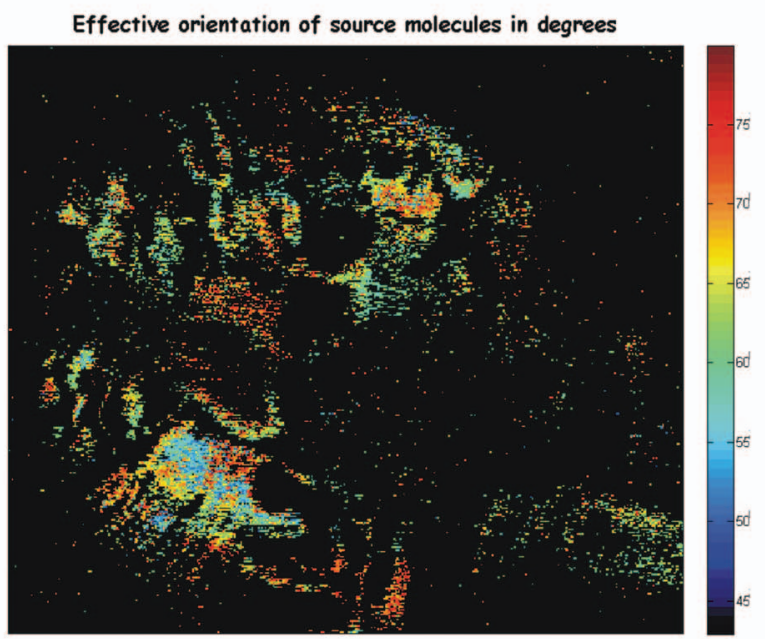

(f)

Fig. 5 (a) Average intensity of nine different PSHG images (each step in the incoming linear polarization is 20 deg) of the C. elegans terminal (posterior) lobe of the pharynx. Scale bar is $10 \mu \mathrm{m}$. This image corresponds to a longitudinal section of the lobe where the grinder is located. The posterior of the worm is upper left and the anterior is lower right. (b) Coefficient of determination ( $\left.r^{2}\right)$. (c) $a=\chi_{31}^{(2)} / \chi_{15}^{(2)}$ and (d) $b=\chi_{33}^{(2)} / \chi_{15}^{(2)}$ are obtained ratios between the elements of the $\chi^{(2)}$ tensor. (e) Orientation of thick filaments $(\phi)$. Arrows indicate real inclination and their colors represent the intervals of the degree color map on the top. The white arrow indicates the position of the grinder system. (f) Harmonophore orientation $\left(\theta_{e}\right)$ in degrees. 


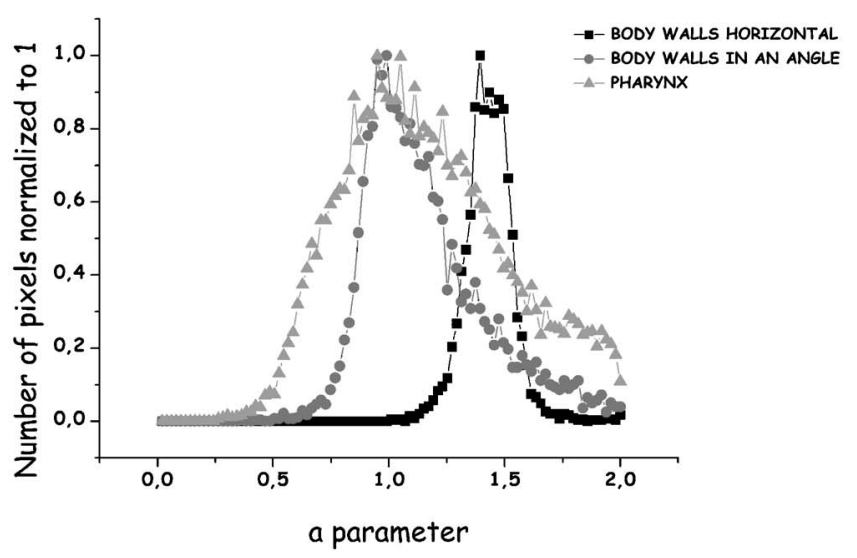

(a)

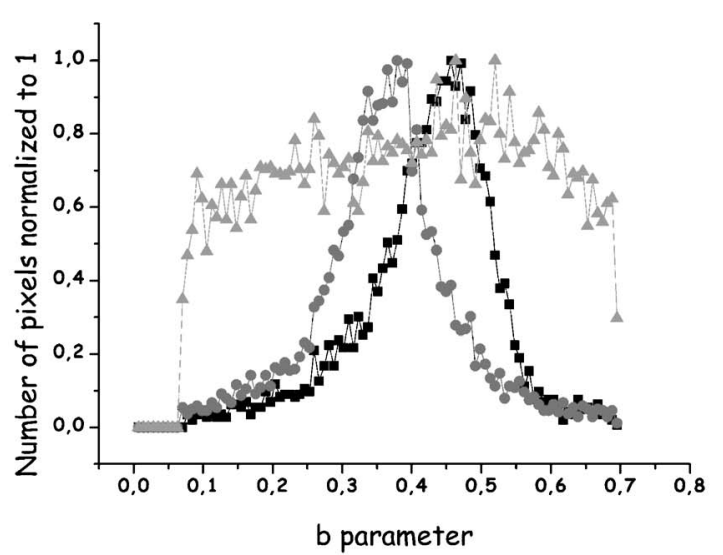

(b)

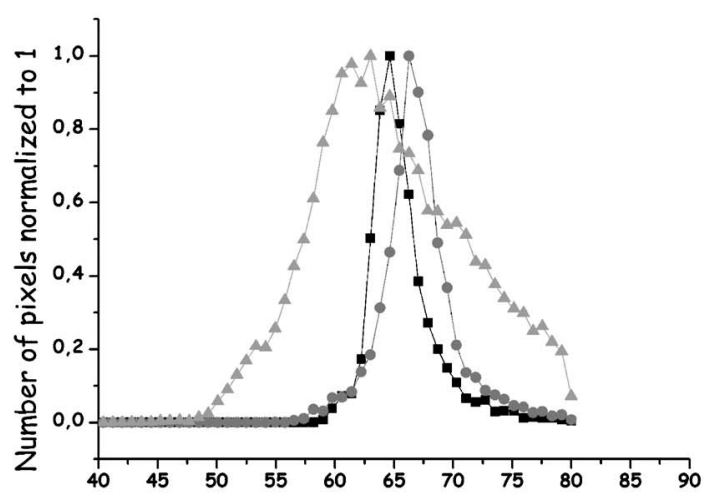

Effective orientation of source molecules in degrees

(c)

Fig. 6 Frequency distribution for the retrieved parameters: (a) a $=\chi_{31}^{(2)} / \chi_{15}^{(2)}$, (b) $b=\chi_{33}^{(2)} / \chi_{15}^{(2)}$, and (c) harmonophores orientation $\left(\theta_{e}\right)$ corresponding to Figs. 3-5.

functionality of a complex muscular system was made apparent. Moreover, it uncovered several parts of muscular cells that are part of the pumping system of the nematode. This contrast was absent in the intensity-only SHG image.

In conclusion, this study has shown that pixel-resolution fitting of PSHG imaging to a biophysical model can work as a filtering (contrast) mechanism using the coefficient of determination $\left(r^{2}\right)$, giving complementary information to common intensity SHG microscopy. The demonstration of this achieved advantage in vivo is a first step for the application of PSHG in biomedical imaging in which the different parameter distributions can be used to quantify structural information. This could be of interest, for example, in the diagnosis of muscular dystrophy or other muscular degenerative diseases.

\section{Acknowledgments}

We would like to thank Dr. Joe Culotti from the Samuel Lunenfeld Research Institute in Toronto, who provided us with the C. elegans strain, and Manoj Mathew for his invaluable help and comments on the present work. This work is supported by the Generalitat de Catalunya and by the Spanish government grant TEC2006-12654 SICO. S. Santos acknowledges funding from the Centre Innovacio i Desenvolupament Empresarial-CIDEM (RDITSCON07-1-0006). This research has been partially supported by Fundació Cellex.

\section{References}

1. P. J. Campagnola and L. M. Loew, "Second-harmonic imaging microscopy for visualizing biomolecular arrays in cells, tissues and organisms," Nat. Biotechnol. 21(11), 1356-1360 (2003).

2. W. R. Zipfel, R. M. Williams, and W. W. Webb, "Nonlinear magic: multiphoton microscopy in the biosciences," Nat. Biotechnol. 21(11), 1369-1377 (2003).

3. J. Mertz, "Molecular photodynamics involved in multi-photon excitation fluorescence microscopy," Eur. Phys. J. D 3, 53-66 (1998).

4. P. J. Campagnola, A. C. Millard, M. Terasaki, P. E. Hoppe, C. J. Malone, and W. A. Mohler, "Three-dimensional high-resolution second harmonic generation imaging of endogenous structural proteins in biological tissues," Biophys. J. 81, 493-508 (2002).

5. S. Roth and I. Freund, "Second harmonic generation in collagen," $J$. Chem. Phys. 70, 1637-1643 (1979).

6. I. R.-Mendoza, D. R. Yankelevich, M. Wang, K. M. Reiser, C. W. Frank, and A. Knoesen, "Sum frequency vibrational spectroscopy: the molecular origins of the optical second-order nonlinearity of collagen," Biophys. J. 93, 4433-4444 (2007).

7. P. Stoller, K. M. Reiser, P. M. Celliers, and A. M. Rubenchik, "Polarization-modulated second harmonic generation in collagen," Biophys. J. 82, 3330-3342 (2002).

8. L. Gao, L. Jin, P. Xue, J. Xu, Y. Wang, H. Ma, and D. Chen, "Reconstruction of complementary images in second harmonic generation microscopy," Opt. Express 14, 4727-4735 (2006).

9. C. Odin, Y. Le Grand, A. Renault, L. Gailhouste, and G. Baffet, "Orientation fields of nonlinear biological fibrils by second harmonic generation microscopy," J. Microsc. 229, 32-38 (2008).

10. Y. Guo, P. P. Ho, A. Tirksliunas, F. Liu, and R. R. Alfano, "Optical harmonic generation from animal tissues by the use of picosecond and femtosecond laser pulses," Appl. Opt. 35, 6810-6813 (1996).

11. M. Kobayashi, K. Fujita, T. Kaneko, T. Takamatsu, O. Nakamura, and S. Kawata, "Second-harmonic-generation microscope with a microlens array scanner," Opt. Lett. 27, 1324-1326 (2002).

12. T. Boulesteix, E. Beaurepaire, M. P. Sauviat, and M. C. SchanneKlein, "Second-harmonic microscopy of unstained living cardiac myocytes: measurements of sarcomere length with 20-nm accuracy," Opt. Lett. 29, 2031-2033 (2004)

13. C. Greenhalgh, N. Prent, C. Green, R. Cisek, A. Major, B. Stewart, and V. Barzda, "Influence of semicrystalline order on the secondharmonic generation efficiency in the anisotropic bands of myocytes," Appl. Opt. 46, 1852-1859 (2007).

14. S. W. Chu, S. Y. Chen, G. W. Chern, T. H. Tsai, Y. C. Chen, B. L. Lin, and C. K. Sun, "Studies of $\chi^{(2)} / \chi^{(3)}$ tensors in submicron-scaled bio-tissues by polarization harmonics optical microscopy," Biophys. J. 86, 3914-3922 (2004)

15. M. Both, M. Vogel, O. Friedrich, F. Wegner, T. Kunsting, R. H. A. Fink, and D. Uttenweiler, "Second harmonic imaging of intrinsic signals in muscle fibers in situ." J. Biomed. Opt. 9, 882-892 (2004).

16. M. Vogel, S. Schurmann, O. Friedrich, F. Wegner, M. Both, and R. H. A. Fink, "Scanning multi photon microscopy of SHG signals from 
single myofibrils of mammalian skeletal muscle," Proc. SPIE 6089, 247-253 (2006).

17. S. V. Plotnikov, A. C. Millard, P. J. Campagnola, and W. A. Mohler, "Characterization of the myosin-based source for second harmonic generation from muscle sarcomeres," Biophys. J. 90, 328-339 (2006).

18. F. Tiaho, G. Recher, and D. Rouede, "Estimation of helical angles of myosin and collagen by second harmonic generation imaging microscopy," Opt. Express 15, 12286-12295 (2007).

19. C. K. Chou, W. L. Chen, P. T. Fwu, S. J. Lin, H. S. Lee, and C. Y. Dong, "Polarization ellipticity compensation in polarization secondharmonic generation microscopy without specimen rotation," $J$. Biomed. Opt. 13, 014005-7 (2008).

20. M. E. Llewellyn, R. P. J. Barretto, S. L. Delp, and M. J. Schnitzer, "Minimally invasive high-speed imaging of sarcomere contractile dynamics in mice and humans," Nature 454, 784-788 (2008).

21. S.-W. Chu, S.-P. Tai, C.-K. Sun, and C.-H. Lin, "Selective imaging in second-harmonic generation microscopy by polarization manipulation," Appl. Phys. Lett. 91, 103903 (2007)

22. S. W. Chu, S. Y. Chen, T. H. Tsai, T. M. Liu, C. Y. Lin, H. J. Tsai, and C. K. Sun, "In vivo developmental biology study using noninvasive multi-harmonic generation microscopy," Opt. Express 11, 3093-3099 (2003).

23. D. A. Dombeck, K. A. Kasischke, H. D. Vishwasrao, M. Ingelsson, B. T. Hyman, and W. W. Webb, "Uniform polarity microtubule assemblies imaged in native brain tissue by second-harmonic generation microscopy," Proc. Natl. Acad. Sci. U.S.A. 100, 7081-7086 (2003).

24. A. C. Kwan, D. A. Dombeck, and W. W. Webb, "Polarized microtubule arrays in apical dendrites and axons," Proc. Natl. Acad. Sci. U.S.A. 105, 11370-11375 (2008)

25. G. C. Cox, N. Moreno, and J. Feijo, "Second harmonic imaging of plant polysaccharides," J. Biomed. Opt. 10, 024013 (2005).

26. I. Amat-Roldán, I. G. Cormack, P. Loza-Alvarez, and D. Artigas, "Starch-based second-harmonic-generated collinear frequencyresolved optical gating pulse characterization at the focal plane of a high-numerical-aperture lens," Opt. Lett. 29(19), 2282-2284 (2004).

27. A. K. N. Thayil, E. J. Gualda, S. Psilodimitrakopoulos, I. G. Cormack, I. Amat-Roldan, M. Mathew, D. Artigas, and P. Loza-Alvarez, "Starch-based backwards SHG for in-situ MEFISTO pulse characterization in multiphoton microscopy," J. Microsc. 230(1), 70-75 (2008).

28. R. M. J. Brown, A. C. Millard, and P. J. Campagnola, "Macromolecular structure of cellulose studied by second-harmonic generation imaging microscopy," Opt. Lett. 28, 2207-2209 (2003).

29. O. Nadiarnykh, R. B. LaComb, P. J. Campagnola, and W. A. Mohler, "Coherent and incoherent SHG in fibrillar cellulose matrices," Opt. Express 15, 3348-3360 (2007).

30. I. Freund, M. Deutsch, and A. Sprecher, "Connective tissue polarity: optical second harmonic microscopy, crossed-beam summation, and small-angle scattering in rat tail tendon," Biophys. J. 50, 693-712 (1986).

31. L. Moreaux, O. Sandre, and J. Mertz, "Membrane imaging by second-harmonic generation microscopy," J. Opt. Soc. Am. B 17, 1685-1694 (2000).

32. L. Moreaux, O. Sandre, S. Charpak, M. Blanchard-Desce, and J. Mertz, "Coherent scattering in multi-harmonic light microscopy," Biophys. J. 80, 1568-1574 (2001).

33. Z. F. Altun and D. H. Hall Eds., Muscular System in WormAtlas, 2002-2006, http://www.wormatlas.org.

34. I. A. Telley and J. Denoth, "Sarcomere dynamics during muscular contraction and their implications to muscle function," J. Muscle Res. Cell Motil. 28, 89-104 (2007).

35. H. Kagawa, T. Takaya, R. Ruksana, F. Anokye-Danso, M. Z. Amin, and H. Terami, "C. elegans model for studying tropomyosin and troponin regulations of muscle contraction and animal behavior," Adv. Exp. Med. Biol. 592, 153-161 (2007).

36. H. F. Epstein, D. M. Miller, I. Ortiz, and G. C. Berliner, "Myosin and paramyosin are organized about a newly identified core structure," $J$. Cell Biol. 100, 904-915 (1985).

37. J. M. Mackenzie and H. F. Epstein, "Paramyosin is necessary for determination of nematode thick filament length in vivo," Cell $\mathbf{2 2}$, 747-755 (1980).

38. M. F. Schmid and H. F. Epstein, "Muscle thick filaments are rigid coupled tubules, not flexible ropes," Cell Motil. Cytoskeleton 41, 195-201 (1998).

39. T. Gutsmann, G. E. Fantner, M. Venturoni, A. Ekani-Nkodo, J. B. Thompson, J. H. Kindt, D. E. Morse, D. K. Fygenson, and P. K. Hansma, "Evidence that collagen fibrils in tendons are inhomogeneously structured in a tubelike manner," Biophys. J. 84, 2593-2598 (2003).

40. S. Brenner, "The genetics of Caenorhabditis elegans," Genetics 77, 71-94 (1974).

41. R. W. Boyd, Nonlinear Optics, Academic Press, San Diego (1992).

42. A. Leray, L. Leroy, Y. Le Grand, C. Odin, A. Renault, V. Vie, D. Rouede, T. Mallegol, O. Mongin, M. H. V. Werts, and M. BlanchardDesce, "Organization and orientation of amphiphilic push-pull chromophores deposited in Langmuir-Blodgett monolayers studied by second-harmonic generation and atomic force microscopy," Langmuir 20, 8165-8171 (2004).

43. V. Le Floc'h, S. Brasselet, J.-F. Roch, and J. Zyss, "Monitoring of orientation in molecular ensembles by polarization sensitive nonlinear microscopy," J. Phys. Chem. B 107, 12403-12410 (2003).

44. G. L. Marquez, L. V. Wang, S.-P. Lin, J. A. Schwartz, and S. L. Thomsen, "Anisotropy in the absorption and scattering spectra of chicken breast tissue," Appl. Opt. 37, 798-804 (1998).

45. G. J. Simpson and K. L. Rowlen, "An SHG magic angle: dependence of second harmonic generation orientation measurements on the width of the orientation distribution," J. Am. Chem. Soc. 121, 26352636 (1999). 